\title{
1 Number neurons in the nidopallium of young domestic chicks
}

2 Dmitry Kobylkov ${ }^{1}$, Uwe Mayer ${ }^{1}$, Mirko Zanon ${ }^{1}$, Giorgio Vallortigara ${ }^{1 *}$

$3{ }^{1}$ Center for Mind/Brain Science, University of Trento, Rovereto, 38068, Italy.

$4 \quad$ * Corresponding author: Giorgio Vallortigara

$5 \quad$ P.zza Manifattura, 1, 38068 Rovereto, Italy; tel. 0464808676

6 email: giorgio.vallortigara@unitn.it

7

8 Classification

9 Major category: Biological sciences

10 Minor categories: Psychological and Cognitive Sciences, Neuroscience

11 Keywords: NCL, numerosity, electrophysiology, birds, evolution 


\section{Abstract}

22 Numerical cognition is ubiquitous in the animal kingdom. Domestic chicks are a 23 widely used developmental model for studying numerical cognition. Soon after 24 hatching, chicks can perform sophisticated numerical tasks. Nevertheless, the neural 25 basis of their numerical abilities has remained unknown. Here, we describe for the 26 first time number neurons in the caudal nidopallium (functionally equivalent to the 27 mammalian prefrontal cortex) of young domestic chicks. Number neurons that we 28 found in young chicks showed remarkable similarities to those in the prefrontal 29 cortex and caudal nidopallium of adult animals. Thus, our results suggest that 30 numerosity perception based on the labeled-line code provided by number neurons might be an inborn feature of the vertebrate brain.

\section{Significance}

34 Numerosity, i.e. the number of items in a set, is a significant aspect in the perception 35 of the environment. Behavioural and in silico experiments suggest that number sense belongs to a core knowledge system and can be present already at birth. However, neurons sensitive to the number of visual items have been so far

38 described only in the brain of adult animals. Therefore, it remained unknown to what 39 extent their selectivity would depend on visual learning and experience. We found 40 number neurons in the caudal nidopallium (a higher associative area with functional 41 similarities to the mammalian prefrontal cortex) of very young, numerically naïve 42 domestic chicks. This result suggests that numerosity perception is possibly an 43 inborn feature of the vertebrate brain. 


\section{Introduction}

45 Be it a number of conspecifics in a group (Balestrieri et al. 2019), a number of food

46 items (Hunt et al. 2008), or a number of motifs in a song (Templeton et al. 2005),

47 correct estimation of quantities is of vital importance for animals. Several behavioural

48 studies have confirmed that numerical competence is not a prerogative of human

49 beings, but is a widespread phenomenon in the animal kingdom (reviewed by Nieder

50 2019, Lorenzi et al. 2021). Mammals (Davis and Albert 1986, Ward and Smuts 2007,

51 Beran et al. 2008), birds (Lyon 2003, Templeton et al. 2005, Rugani et al. 2009),

52 reptilians (Gazzola et al. 2018), amphibians (Stancher et al. 2015), fishes (Potrich et

53 al. 2015), and invertebrates (Bortot et al. 2021), although evolutionary distant, all can

54 spontaneously assess quantities using an approximate number system (Brannon

55 and Merritt 2011).

56 For the approximate number system, which is based on the Weber-Fechner law

57 (Nieder 2016), the perception of cardinal numbers resembles the perception of

58 continuous physical stimuli. As a consequence, discrimination of quantities is

59 imprecise and depends on the numerical distance between stimuli. In other words, it

60 is easier to tell apart 5 and 10 than 9 and 10 . Moreover, discrimination of quantities

61 becomes increasingly difficult with the numerical size. For a given numerical distance

62 (e.g., 1) it is easier to discriminate between numbers with low magnitudes (1 vs. 2),

63 than with high magnitudes (9 vs. 10).

64 Recent research has uncovered that the approximate number system relies on the

65 activity of a specific neuronal population. Neurons that respond to abstract

66 numerosity irrespective of objects' physical appearance (shape, colour, size) have

67 been found in the forebrain of human and non-human primates (Nieder 2012, Kutter 
et al. 2018) and in crows (Ditz and Nieder 2015). In mammals, numerical responses were recorded in the parietal and the prefrontal cortices (PFC, Nieder 2012). In birds, similar neurons have been described in the caudolateral nidopallium (NCL, Ditz and Nieder 2015). The NCL is believed to be an analogue of the PFC in the avian brain (Güntürkün et al. 2021) and is involved in a variety of cognitive processes, including memory formation (Diekamp et al. 2002, Hahn et al. 2021), abstract rule learning (Veit and Nieder 2013), and action planning (Veit et al. 2015).

Both monkeys and crows are among the most evolutionary advanced species of their phylogenetic groups. They independently developed sophisticated intellectual capacities (Nieder et al. 2020) and both possess enlarged forebrains (Olkowicz et al., 2016). The neural representation of numerosities described in these species also shares remarkable similarities (Nieder et al. 2002, Ditz and Nieder 2015, Viswanathan and Nieder 2013, Wagener et al. 2018). In both species, the number neurons tuned to a preferred numerosity show a gradual decrease in firing rate to other numerosities (numerical distance effect). Their tuning curves are skewed towards larger numerosities and become progressively broader (less selective) with 84 increasing numerosities (numerical size effect). However, it is unclear whether the presence of similar number neurons in these two species emerges as a consequence of their elaborate cognitive skills and enlarged forebrains. To understand the evolution of the number sense we need to explore its neural correlates in distant bird species with more ancestral traits.

Moreover, until now, number neurons have been described only in adult animals

90 (e.g. Nieder et al. 2002, Sawamura et al. 2002, Viswanathan and Nieder 2013, Ditz 91 and Nieder 2015, Wagener et al. 2018). At the same time, behavioural data from 92 human infants (Izard et al. 2009) and young domestic chicks (Rugani et al. 2008, 
93 2009) indicate that some core numerical abilities might be an inborn or

94 spontaneously emerging (Kim et al. 2021, Nasr et al. 2019) property of the

95 vertebrate brain. Testing the presence of number neurons in young and untrained

96 organisms is crucial to verify this hypothesis.

97 In our study, we aimed to describe the neural correlates of the number sense in 98 domestic chicks (Gallus gallus), which belong to a sister group of modern Neoaves 99 (Prum et al. 2015). The domestic chick is a well-established developmental model for 100 studying numerical cognition. Soon after hatching these birds are already capable of 101 discriminating quantities (Rugani et al. 2008, 2013) and even performing basic 102 arithmetic operations (Rugani et al. 2009). It has also been shown that young chicks 103 represent numbers across the mental number line (Rugani et al. 2015), a cognitive 104 ability that had been previously attributed only to humans.

105 We hypothesised that neural processing of numerical information in young untrained 106 chicks might be similar to crows, despite them having been evolving independently over the last $~ 70$ million years (Prum et al. 2015). In a domestic chicken, the NCL is morphologically different from that of corvids (von Eugen et al. 2020), but it is unclear whether this reflects any functional difference. Therefore, we decided to search for neural responses to numerical stimuli in the NCL of domestic chicks. For this purpose we habituated young chicks to a computer monitor, where numerical stimuli were presented (Fig. 1A). We explored neural responses to numerosities from 1 to 5 .

113 To control for non-numerical parameters we presented three different categories of stimuli:“radius-fixed", "area-fixed”, and "perimeter-fixed” (Fig. 1B). 


\section{Results}

118 We recorded the activity of 471 units in the NCL of young domestic chicks and 119 examined the mean firing rate of each unit during stimulus presentation (Fig. 2A-B).

120 Among these units, $53(11 \%)$ responded to numerosity irrespective of the stimulus 121 type (henceforth "number neurons", see Nieder 2016). This was revealed by a twoway ANOVA with the factors "numerosity" (5 levels: 1-5) and "stimulus type" (3 levels: "radius-fixed", "area-fixed", "perimeter-fixed"). The unit was considered as a

124 number neuron only if the main effect of the factor "numerosity" was highly significant $125(p<0.01)$, but not the effect of the "stimulus type" or the interaction between the two 126 factors. The numerosity that elicited the strongest neural response was defined as 127 the preferred numerosity for this number neuron (after Ditz and Nieder 2015). Five examples of number neurons tuned to different numerosities are shown in Fig. 2C-G. The corresponding statistics are summarised in Table 1. The responses of all 53 number neurons are summarised in Fig. 3A (for statistical results see Table S1 in the supplementary materials, for an example of trials with numerical responses see Video S1 in the supplementary materials).

Most of the number neurons were tuned to the numerosity $1(30 \%, N=16)$ and 5 $134(32 \%, N=17)$. However, we found neurons responsive to other numerosities as well (2: $11 \%, N=6 ; 3: 9 \%, N=5 ; 4: 17 \%, N=9$ ), covering the whole range of tested numerosities (Fig. 2B).

When grouped by their preferred numerosity, the tuning curves of number neurons were asymmetric and increasingly wider towards larger numerosities (Fig. 3B). To quantify this numerical magnitude effect, we plotted neural filter functions of single neurons on four different scales: a linear scale, a power function with an exponent of 
1410.5 , a power function with an exponent of 0.33 , and a logarithmic (log2) scale. The

142 neural filter functions became significantly more symmetric on a non-linear scale

143 (Friedman test: $X^{2}(3)=10.653 ; p=0.014$, Fig. 4A), with the linear scale significantly

144 different from the power 0.33 and logarithmic scales (Nemenyi test: $p=0.022$ and $p$

$145=0.028$, respectively), but not from the power 0.5 scale (Nemenyi test: $p=0.176$ ).

146 The sigma of the Gaussian fit increased with numerosity only when plotted on a

147 linear scale (Fig. 4B, slope of the linear fit $=0.17$ ) but not with other non-linear scales

148 (Fig. 4B, power 0.5: slope $=-0.003$, power 0.33: slope $=-0.01$, log2: slope $=-0.03$ )

To evaluate the probability of finding false positive numerical responses in our dataset, we used the trial-shuffle method (1000 times for every recorded unit). After each shuffling we selected false-positive number neurons by the same statistical criteria described above. The proportion of real number neurons (11\%; 53 out of 471) was significantly higher than the proportion of false-positive units $(0,95 \% ; 4488$ out of 471000 ) obtained by the analysis of randomly shuffled trials (proportion test: $\left.X^{2}(1)=512,56, p<0.001\right)$

We further compared the tuning curves between the false positive number neurons and the real number neurons. For this, for each numerical category we randomly selected the same number of corresponding false-positive neurons as the actually recorded number neurons in that category (Fig 2B). We compared the tuning curves of the real and false-positive neurons (Fig 4C) computing a two-way ANOVA with the factors "absolute numerical distance" (5 levels: 0-4) and "data type" (2 levels: real/false positive). The response of real neurons decreased gradually with numerical distance, meaning that closest numerosities were more likely to trigger the number neurons. In contrast, the tuning curve of the false-positive neurons, which by 
markedly different from real number neurons (interaction for the factor "numerical distance * data type": $\left.F_{(1,526)}=6.275 ; p=0.01\right)$.

We further described the selectivity of number neurons for unique numerosities by performing a post hoc analysis and comparing the response between the most preferred and other numerosities (Table 2). 9 out of 53 number neurons showed statistically different firing rates between the most-preferred and closest neighbouring numerosities. As expected from the numerical size effect, neurons tuned to lower numerosities are generally more selective than neurons coding for larger numerosities. 6 out of 16 neurons with the preferred numerosity 1 significantly decreased their firing rate in response to the numerosity 2. At the same time, out of 17 neurons that preferentially responded to the numerosity 5 , only 1 neuron showed significantly lower response to the numerosity 4 . The number neurons generally better differentiated between numerosities with increasing numerical distance (GLM for absolute numerical distance: $X^{2}(18)=3.935, p=0.047$; GLM for the log of the numerical distance: $X^{2}(18)=6.544, p=0.011$ ). This effect was more prominent when the numerical distance was calculated on the logarithmic scale: the GLM for the log of the numerical distance had a better fit than the GLM for absolute numerical distance $(\triangle \mathrm{BIC}=6.36)$. For the summary of all post hoc results, see TableS2 in the supplementary materials.

\section{Discussion}

We recorded number neurons in 8-12 day old chicks, the youngest animals in which number neurons have been described so far. In the NCL of young domestic chicks, $11 \%$ of neurons showed a strong selective response to numerical stimuli, confirming 
190 the role of this structure in avian numerical cognition. Moreover, our chicks were not

191 trained in any numerical discrimination task. Instead, they were simply paying

192 attention to the screen, where the numerical stimuli were presented (passive fixation,

193 Hussar and Pasternak 2009). Thus, our young chicks can be considered numerically

194 naïve (see Viswanathan and Nieder 2013).

195 This result is in line with previous studies on numerically naïve adult animals of

196 distant lineages: $14 \%$ of neurons in the prefrontal cortex (PFC) of adult monkeys

197 (Viswanathan and Nieder 2013) and 12\% of neurons in the NCL of adult crows

198 (Wagener et al. 2018) have shown numerical response. It is important to note that in

199 any of these studies including our own, we cannot completely exclude a potential

200 unsupervised learning effect due to a repeated exposure to numerical stimuli.

201 Indeed, recent studies have found that in a deep neural network designed to analyse

202 images, a similar amount of number detectors can emerge spontaneously (Nasr et

203 al. 2019), even without pre-training (Kim et al. 2021). However, the hypothesis that

204 number neurons can spontaneously emerge in the biological visual system has not

205 been directly tested so far.

206 Our data, together with behavioural evidence from newly hatched domestic chicks

207 (Rugani et al. 2008, 2009) and newborn infants (Izard et al. 2009), suggest that

208 numerosity detection might be an inborn or spontaneously emerging feature of the

209 brain. Our results certainly should not be automatically extrapolated to other

210 newborn organisms, since the domestic chicken is a precocial species with a very

211 rapid development after hatching. Nevertheless, even in 2-weeks-old chicks, the

212 functional organization of the brain still remains immature and flexible (Zappia and

213 Rogers 1987). Thus, the presence of number neurons in young chicks, which were

214 not trained to discriminate any specific numerosity, supports the idea of an inborn 
215 number sense. Moreover, since behavioural studies (Rugani et al. 2009, 2013) show

216 that newly hatched chicks perceive numerical information, we might expect to find

217 number neurons already at the first day after hatching.

218 The number neurons in domestic chicks showed very similar features to those 219 observed in primates and crows (Viswanathan and Nieder 2013, Ditz and Nieder $2202015,2016)$. First, the number neurons we observed were tuned to specific 221 numerosities, in accordance with the labelled-line code shown for the number 222 neurons in the NCL of crows (Ditz and Nieder 2015, 2016). Second, chicks' number 223 neurons showed a specific decay in response to non-preferred numerosities, which 224 was similar to number neurons in other species and could not have been obtained by 225 chance (see false-positive neurons in Fig. 4C). Third, we observed the numerical 226 magnitude effect: the tuning curves of number neurons became wider with increased 227 numerosity and more symmetrical when plotted on the non-linear scale (Fig. 3B). 228 Also the selectivity of the neural response to non-preferred numerosities increased 229 with the logarithm of the numerical distance, rather than with the absolute numerical 230 distance.

231 The overall low selectivity to specific numerosity at a single-cell level, as revealed by 232 the post hoc analysis (Table 2), appears to be similar to what was observed in other 233 species (Viswanathan and Nieder 2013; Ditz and Nieder 2015, 2016). How brains 234 can depict precise numerosities with such a noisy system may be explained by a 235 population rate code (Nieder 2016). Single cells respond to every trial in a 236 probabilistic way and only on average show increased firing rates to a given 237 numerosity. Smooth tuning curves only emerge after the neural filter functions of 238 many single cells tuned to the same numerosity are pooled together (Fig. 3B). For an 239 animal to immediately assess numerosity, independent responses of several number 
neurons should be averaged simultaneously over a large population. Thus, at the

neural population level, stimulation by a specific numerosity would result in a distinct

244 Given these striking similarities between number neurons in the NCL of crows and

245 chicks, it seems a reasonable guess that they share the same evolutionary origin.

246 This, in turn, would mean that the neural mechanism of the number sense is not an

247 advanced evolutionary adaptation of a few highly intelligent species. Number

248 neurons in the NCL are likely an ancestral feature in avian species, since domestic

249 chicks belong to the sister group of modern Neoaves (Prum et al. 2015).

While it is very tempting to go one step further and discuss the idea of the common evolutionary origin of number neurons for all vertebrates, this would be too speculative. The NCL in birds and the prefrontal cortex in mammals do share similar functions, but are not homologous structures (Güntürkün and Bugnyar 2016, Preuss and Wise 2021). Birds independently developed cortical brain regions, including the NCL, that enabled their high cognitive functions (Güntürkün et al. 2021) based on similar to mammalian pallial architecture (Stacho et al. 2020). The same is likely true also for the Dc (dorsal-central) region in the telencephalon of zebrafish, which has been recently shown to process numerical information (Messina et al. 2021). The possibility of a direct homology between the Dc and either the PFC or the NCL can only be addressed at a macroscopic level. These three regions likely belong to larger neocortex homologues in the respective species (Briscoe and Ragsdale 2019, but see Puelles 2017). However, given the developmental and morphological differences between these areas, new cortical subregions with similar functions must have appeared multiple times during independent evolution of these structures. 
265 Two hypotheses can be put forward on the evolution of number sense in vertebrates.

266 The first one is that number perception evolved independently several times in

267 different phylogenetic groups, although our data strongly suggest that number

268 neurons in the nidopallium are an ancestral trait of birds. This mechanism, however,

269 still can be an adaptation that evolved in parallel to mammalian number neurons. In

270 this case, the identical labelled-line coding scheme for numerosities adopted by birds

271 and mammals might be computationally advantageous and, therefore, evolved

272 independently in both groups (Ditz and Nieder 2015). The second hypothesis is that

273 numerosity processing can be based upon an ancient core neural circuit shared

274 among all vertebrates. In this case, we would expect to find numerosity responses in

275 other, evolutionary conserved brain regions homologically shared among vertebrates

276 (Lorenzi et al. 2021). Indeed, some indirect evidence suggests that at least a coarse

277 estimation of quantity, i.e. more vs. less, might be present already at the subcortical

278 level in humans (Collins et al. 2017) and in the midbrain of birds (Gusel'nikov et al.

279 1971) and zebrafish (Preuss et al. 2014).

280 These hypotheses are, however, not mutually exclusive. The putative ancestral 281 neural circuit might be dedicated to assess continuous physical parameters normally 282 associated with the numerosity, like total area of the stimulus. On the contrary, 283 higher order brain processing leading to estimation of cardinal numerosities at a 284 more abstract level may have developed several times, together with the 285 independent evolution of new cortex homologue brain areas in distant phylogenetic 286 groups (Striedter and Northcutt 2019).

287 Summing up, our study provides the first step in addressing a complex evolutionary 288 and developmental aspect of numerical cognition. By implementing a simplified 289 training procedure, we could, for the first time, demonstrate the existence of number 
290

291

292

293

294

295

296

297

298

299

neurons in young numerically naïve domestic chicks. In the future, this method might be easily adopted for studying the neural correlates of numerical cognition in other brain regions, as well as in other species.

\section{Materials and methods}

\section{Subjects}

12 domestic chicks (Gallus gallus domesticus) of both sexes from the Aviagen ROSS 308 strain were used. Fertilized eggs were obtained from a local commercial hatchery (CRESCENTI Società Agricola S.r.l.-AllevamentoTrepola-cod. Allevamento 127BS105/2). Eggs were incubated and hatched within incubators (Marans P140TU-P210TU) at a temperature of $37.7^{\circ} \mathrm{C}$, with $60 \%$ humidity in a dark room. After hatching in dark incubators, chicks were isolated and housed individually in metal cages (28 cm wide $\square \times \square 32 \mathrm{~cm}$ high $\square \times \square 40 \mathrm{~cm}$ deep) with food and water available ad libitum, at a constant room temperature of $30-32{ }^{\circ} \mathrm{C}$ and a constant light-dark regime of $14 \mathrm{~h}$ light and $10 \mathrm{~h}$ dark. All experimental protocols were approved by the research ethics committee of the University of Trento and by the Italian Ministry of Health (permit number 745/2021-PR).

\section{Experimental setup}

The setup consisted of a rectangular shaped arena (28 X $40 \times 32 \mathrm{~cm}$; W X L X H) with metal walls that were grounded. In the centre of one of the shorter walls there was a circular opening (diameter $12 \mathrm{~cm}$ ). A computer screen (AOC AGON AG271QG4, 144Hz) used for stimuli presentation was positioned directly behind the circular opening. Within the rectangular arena was a small wooden box $14 \times 13 \times 22$ 
$313(\mathrm{~W} X \mathrm{X} \times \mathrm{H}) \mathrm{cm}$ whose frontal wall was made of a metal grid in. The box was placed

$31425 \mathrm{~cm}$ in front of the circular opening with the screen (Fig. 1A). During the 315 experiments chicks remained inside the box from where they could observe the 316 stimuli. Stimulus presentation was controlled by the PsychoPy toolbox (Pierce et al. 317 2019).

\section{Habituation procedure}

319 The habituation occurred between the $3^{\text {rd }}$ and the $6^{\text {th }}$ day after hatching. On the $3^{\text {rd }}$ day post hatching, chicks learned to peck on mealworms. During the day 4 after hatching, chicks were, first, habituated to the experimental setup and then to the number stimuli appearing on the screen. The birds received mealworms every time after the stimulus appeared on the screen, which motivated them to pay attention to 324 the moment when any stimulus would appear. The stimuli were presented and 325 rewarded randomly, so that chicks would not associate any particular numerosity 326 with the reward. During the days 5 and 6 post hatching we gradually decreased the 327 reward rate, so that birds would still pay attention to the screen even without getting 328 a mealworm. This procedure allowed us to minimize rewarding during actual recording sessions.

\section{Surgery and recordings}

331 On the $7^{\text {th }}$ day after hatching chicks were fully anaesthetized using Isoflurane 332 inhalation (1.5 - 2.0\% gas volume, Vetflurane, $1000 \mathrm{mg} / \mathrm{g}$, Virbac, Italy) and placed in 333 the stereotaxic apparatus with a bar fixed at the beaks' base and tilted $45^{\circ}$ to ear 334 bars. Local anaesthesia (Emla cream, 2.5\% lidocaine $+2.5 \%$ prilocaine, 335 AstraZeneka, S.p.A.) was applied to the ears and skull skin before and after the 336 surgery. Metal screws were placed into the skull for grounding and stabilisation of 
337 the implant. A small craniotomy was made in the skull on the right hemisphere above

338 the $\mathrm{NCL}(1.0 \mathrm{~mm}$ anterior to the bregma, $4.5 \mathrm{~mm}$ lateral to the midline). For

339 extracellular recordings we used self-wired tetrodes made out of formvar-insulated

340 Nichrome wires (17.78 $\mu$ m diameter, A-M Systems, USA), which were gold-plated to

341 reduce the impedance to $300-400 \mathrm{kOm}$ (controlled by nanoZ, Plexon Inc., USA).

342 Then, a commercially available Halo-5 microdrive (Neuralynx, USA) was assembled

343 according to the producer instructions, where four single tetrodes were put into

344 polymicro tubes (inner diameter $0.1 \mathrm{~mm}$ ) and glued to the plastic shuttles. The

345 microdrive was implanted and fixed first with quick adhesive silicone (Kwik-Sil, World

346 Precision Instruments, USA) and then with dental cement (Henry Schein Krugg Srl,

347 Italy). To increase the probability of finding number-responsive units, we did not glue

348 the electrode tips within the tetrodes We, thus, considered each tetrode as a brush-

349 like arrangement of four single electrodes. Since in this brush arrangement the

350 positions of the electrode tips can vary, some of the electrodes may have recorded

351 signals from the same neurons. Hence, for sorting and subsequent analyses we

352 chose only the best electrode from each tetrode at every recording position to avoid

353 double-counting of the same neurons.

354 After the surgery, the chicks were left to recover until the next day in their home 355 cages. Between the $8^{\text {th }}$ and the $12^{\text {th }}$ day after hatching we recorded neural 356 responses to numerical stimuli in the NCL of chicks. Before every recording session 357 the microdrive was connected to the Plexon system (Plexon Inc., USA) via a 358 QuickClip connector and an omnetics headstage (Neuralynx, USA). After every 359 recording session the tetrodes were manually advanced by $60-100 \mu \mathrm{m}$.

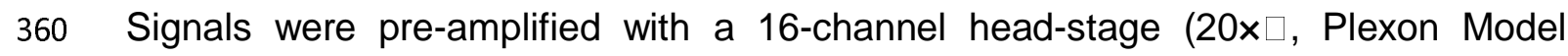
361 number: PX.HST/16V-G20-LN) subsequently amplified $1000 \square \times \square$, digitalised and 
362 filtered $(300 \mathrm{~Hz}$ high-pass filter, $3 \mathrm{kHz}$ low-pass filter and $50 \mathrm{~Hz}$ noise removal).

363 Common average referencing (CAR) method (the averaged signal across channels)

364 of the PlexControl system was used for referencing. Spikes were detected with the

365 PlexControl software with an automatic 4-sigma threshold from the average noise

366 level. Subsequently, spike sorting was performed manually in the Plexon Offline

367 Sorter (see Figure S1 in supplementary information for examples of the raw signal 368 and spike sorting).

\section{Stimuli}

370 As numerical stimuli we used red dots outlined with a thin black line that appeared in

371 the centre of the screen in a white background circle $6 \mathrm{~cm}$ in diameter. The size of

372 stimuli ranged from $0.25 \mathrm{~cm}$ to $1.4 \mathrm{~cm}\left(0.6-3.2^{\circ}\right.$, Schmid and Wildsoet 1998). We

373 explored neural responses to numerosities from 1 to 5 . To control for visual

374 parameters that might interfere with numerosity perception, during every recording

375 session we presented three different types of stimuli (Fig. 1B). "Radius-fixed" type of

376 stimuli consisted of dots with a fixed radius, meaning that area and perimeter

377 increase with numerosity. "Area-fixed" stimuli have constant total area over all

378 numerosities, while the total perimeter of the dots increases with numerosity.

379 "Perimeter-fixed" stimuli have constant total perimeter over all numerosities, while

380 total area of these stimuli decreases with numerosity. To further control that neurons

381 do not respond to other visual parameters except for quantity, the inter-distance

382 interval between dots varied randomly. Moreover, for every day of recording we

383 created a new batch of stimuli consisting of 30 unique images for each

384 numerosity/stimulus type combination. Numerosity stimuli were created using

385 GeNEsIS software (Zanon et al. 2021). 
386 During recording sessions we randomly presented stimuli for 500 ms with 2000 ms

387 of inter-stimulus interval. Experiments were video-recorded using CineLAB system

388 (Plexon Inc., USA). To enhance the motivation of birds to pay attention to the screen, 389 random trials were occasionally rewarded. These trials were subsequently discarded 390 from the analysis.

\section{Histological analysis}

After the last neural recording birds were overdosed with the ketamine/xylazine solution (1:1 ketamine $10 \mathrm{mg} / \mathrm{ml} \square+\square$ xylazine $2 \mathrm{mg} / \mathrm{ml}$ ). Electrolytic lesions were made at the recording sites by applying a high-voltage current to the tetrodes for 1015 seconds. Then, the birds were perfused intracardially with the phosphate buffer (PBS; $0.1 \mathrm{~mol}, \quad \mathrm{pH} \square=\square 7.4, \quad 0.9 \%$ sodium chloride, $\quad 5^{\circ} \mathrm{C}$ ) followed by $4 \%$ paraformaldehyde (PFA). Brains were incubated for at least two days in PFA and further two days in $30 \%$ sucrose solution in PFA. Coronal $60 \mu \mathrm{m}$ brain sections were cut at $\square-20^{\circ} \mathrm{C}$ using a cryostat (Leica CM1850 UV), mounted on glass slides, stained with the Giemsa dye (MG500, Sigma-Aldrich, St. Louis, USA), and cover slipped with Eukitt (FLUKA). Brain sections were examined under the stereomicroscope (Stemi 508, Carl Zeiss, Oberkochen, Germany) to estimate the anatomical position of 403 recording sites.

\section{Data analysis}

405 Based on the analysis of video-recordings, we selected only those trials, when birds were not rewarded. Since in birds there is an almost complete decussation of the optic fibres we recorded from the right hemisphere and we selected only trials, when birds looked at the stimulus with both eyes or with the contralateral (left) eye. For each recorded unit, we excluded trials with a firing rate of less than $1 \mathrm{~Hz}$. For the 
410 final analysis we considered only those units that were recorded for at least 7 trials

411 for each numerosity and stimulus type (on average 23 trials per numerosity/stimulus 412 type).

413 The neural activity of recorded units was analysed as the mean firing rate over 500

414 ms of stimulus presentation. To find numerosity-responsive neurons we performed

415 two-way ANOVA with the numerosity (1 to 5 ) and the stimulus type ("radius-fixed",

416 "area-fixed", "perimeter-fixed") as factors. We considered the recorded neuron as

417 numerosity-responsive if only the effect of the "numerosity" factor was highly

418 significant $(p<0.01)$, but not of the stimulus type or the interaction between the two

419 factors. The numerosity that elicited the strongest neural response was defined as a

420 preferred numerosity for this neuron.

421 For every number neuron we performed a post hoc analysis (Tukey's test) to 422 compare the neural response between the most preferred and other numerosities.

423 To test for the numerical distance effect we applied a GLM for binomial data with the

424 logit-link function. In the model, the proportion of neurons that significantly

425 differentiate between given numerosities was taken as the response variable, and

426 either the absolute numerical distance or the logarithm of the numerical distance was

427 taken as a factor. We then compared the goodness-of-fit of these two models based

428 on the difference in the Bayesian Information Criterion $(\triangle B I C)$, where the lower is the

429 BIC value, the better is the model's fit.

430 To validate the stability of our recordings we performed a cross-validation analysis.

431 For each numerosity-responsive unit we calculated the preferred numerosity for the

432 first and the second half of all trials separately. If the neural response to number

433 stimuli was stable across the recording, we expected the Pearson's correlation 
434 between the first and the second half of the trials to be close to 1 for the whole

435 population of number neurons. The cross-validation analysis showed a strong 436 correlation of $0.82(p<0.001)$ between the preferred numerosity in the first and the 437 second half of the trials, confirming the stability of our recordings.

438 For every number neuron we normalised neural activity by setting the firing rate in 439 response to the preferred numerosity at $100 \%$ and to the least preferred numerosity 440 at $0 \%$. The resulting neural filter functions were averaged by group based on the 441 preferred numerosity, thus, creating numerosity tuning curves for e.g. neurons 442 preferring numerosity 1 , numerosity 2 , etc.

443 To evaluate the chance level of finding false-positive numerical responses in our 444 dataset, for every recorded neuron we shuffled all the trials for 1000 times and 445 performed an ANOVA each time to select false-positive number neurons. We 446 compared the proportion of false-positive and real number neurons with the 447 proportion test. We further compared the tuning curves between the false-positive 448 number neurons and the real number neurons. For this, we randomly sampled from 449 the false-positive neurons the same number of neurons as the one of actually 450 recorded number neurons. We compared the tuning curves of real and false-positive 451 neurons performing a two-factor ANOVA with the interaction of "numerical distance" 452 and "data type" (real/false positive).

453 According to the Weber-Fechner law, the perception of sensory stimuli (including 454 quantities) is proportional to a logarithmic (Fechner 1860) or power scale (Stevens 455 1961) of stimulus magnitude. Therefore, when plotted on a linear scale, one might 456 expect tuning curves to become increasingly asymmetric and wide with increasing 457 numerosity. These properties are usually referred to as a numerical distance effect 
478 European Research Council (ERC) under the European Union's Horizon 2020

and a numerical magnitude effect respectively. To evaluate the symmetry and the width of the neural filter functions we fitted the Gaussian function to the curves (MatLab Curve Fitting Toolbox) plotted on four different scales: linear, a power function with an exponent of 0.5 , a power function with an exponent of 0.33 , and a logarithmic (log2) scale (Ditz and Nieder 2016). The symmetry of the Gaussian fit was estimated based on R-squared $\left(r^{2}\right)$ values, i.e. the higher is $r^{2}$ the better and more symmetric is the fit. The width of the Gaussian fit was reflected by its sigma $(\sigma)$. The four scaling methods were compared based on the $r^{2}$ values by the Friedman's test for non-parametric data with repeated measures with the post-hoc pairwise comparison with the Nemenyi test. The relationship between the numerosity and the sigma of the Gaussian was tested by an ANOVA for different scaling methods separately.

All statistical analyses and visualization of the data was performed in $\mathrm{R}$ ( $\mathrm{R}$ Core Team 2020) with packages "tidyverse", "ggplot2", and "PMCMRplus" and in MATLAB using custom-made scripts and the Curve Fitting Toolbox.

\section{Acknowledgments and funding sources}

We are grateful to Anastasia Morandi-Raikova for her help with handling the chicks. Elena Lorenzi, Andrea Messina, and Matilde Perrino provided very valuable comments to the current manuscript. This work was supported by funding from the research and innovation program (Grant agreement no. 833504 SPANUMBRA) and from a PRIN 2017 ERC-SH4-A (2017PSRHPZ) to G. V. 


\section{Author contributions}

482 D.K, U.M. and G.V. designed the experiment. D.K. and U.M. established the 483 procedure for behavioural training and electrophysiology in freely moving chicks.

484 D.K. performed the experiment and analysed the data. M.Z. developed the numerical 485 stimuli and contributed to apart of the data analysis. D.K. wrote the original draft of 486 the manuscript and implemented the comments of all authors. U.M. and G.V. revised 487 and edited the manuscript.

488 Availability of data and material: A summary table including the mean firing rates 489 for all numerosity-responsive neurons for all trials is included as a supplementary 490 data file. Any additional data that support the findings of this study are available from 491 the corresponding author upon reasonable request.

492 Code availability: The custom codes that were used for data analysis are available 493 from the corresponding author upon reasonable request.

494 Competing financial interests: The authors declare no competing financial 495 interests. 


\section{References}

503 Balestrieri, A., Gazzola, A., Pellitteri-Rosa, D. \& Vallortigara, G. Discrimination of 504 group numerousness under predation risk in anuran tadpoles. Anim. Cogn. 22(2), 505 223-230 (2019). https://doi.org/10.1007/s10071-019-01238-5

506 Beran, M. J., Evans, T. A. \& Harris, E. H. Perception of food amounts by 507 chimpanzees based on the number, size, contour length and visibility of items. Anim. 508 Behav. 75, 1793-1802 (2008). https://doi.org/10.1016/j.anbehav.2007.10.035

509 Bortot, M., Regolin, L. \& Vallortigara, G. A sense of number in 510 invertebrates. Biochem. Biophys. Res. Commun. 564, 37-42 (2021). 511 https://doi.org/10.1016/j.bbrc.2020.11.039

512 Brannon, E. M. \& Merritt, D. J. Evolutionary foundations of the approximate number 513 system. in Space, time and number in the brain (eds. Dehaene, S. \& Brannon, E.M.) 514 207-224 (Academic Press, 2011). https://doi.org/10.1016/B978-0-12-385948$515 \quad 8.00014-1$

516 Briscoe, S. D. \& Ragsdale, C. W. Evolution of the chordate telencephalon. Curr. 517 Biol. 29(13), 647-662 (2019). https://doi.org/10.1016/j.cub.2019.05.026

518 Collins, E., Park, J. \& Behrmann, M. Numerosity representation is encoded in human 519 subcortex. Proc. Natl Acad. Sci. USA 114(14), 2806-2815 (2017). 520 https://doi.org/10.1073/pnas.1613982114

521 Davis, H. \& Albert, M. Numerical discrimination by rats using sequential auditory 522 stimuli. Animal Learning \& Behavior 14(1), $\quad$ 57-59 (1986). 523 https://doi.org/10.3758/BF03200037 
524 Diekamp, B., Kalt, T. \& Güntürkün, O. Working memory neurons in pigeons. J.

525 Neurosci. 22(4), RC210-R210 (2002). https://doi.org/10.1523/JNEUROSCI.22-04-

$526 j 0002.2002$

527 Ditz, H. M. \& Nieder, A. Neurons selective to the number of visual items in the corvid

528 songbird endbrain. Proc. Natl Acad. Sci. USA 112(25), 7827-7832 (2015).

529 https://doi.org/10.1073/pnas. 1504245112

530 Ditz, H. M. \& Nieder, A. Numerosity representations in crows obey the Weber-

531 Fechner law. Proc. R. Soc. B Biol. Sci. 283(1827), 20160083 (2016).

532 https://doi.org/10.1098/rspb.2016.0083

533 Fechner, G. T. Elemente der psychophysik (Vol. 2). (Breitkopf u. Härtel, 1860).

534 Gazzola, A., Vallortigara, G. \& Pellitteri-Rosa, D. Continuous and discrete quantity 535 discrimination in tortoises. Biol. Lett. 14(12), $20180649 \quad$ (2018).

536 https://doi.org/10.1098/rsbl.2018.0649

537 Güntürkün, O. \& Bugnyar, T. Cognition without cortex. Trends Cogn. Sci. 20(4), 291-

538303 (2016). https://doi.org/10.1016/j.tics.2016.02.001

539 Güntürkün, O., von Eugen, K., Packheiser, J. \& Pusch, R. Avian pallial circuits and

540 cognition: A comparison to mammals. Curr. Opin. Neurobiol. 71, 29-36 (2021).

541 https://doi.org/10.1016/j.conb.2021.08.007

542 Gusel'nikov, V. I., Morenkov, É. D. \& Gutsu, I. P. Responses of neurons in the 543 pigeon's optic tectum to visual stimuli. Neurophysiology 3(1), 78-83 (1971). 
544 Hahn, L. A., Balakhonov, D., Fongaro, E., Nieder, A. \& Rose, J. Working memory

545 capacity of crows and monkeys arises from similar neuronal computations. eLife 10,

546 e72783 (2021). DOI: 10.7554/eLife.72783

547 Hunt, S., Low, J. \& Burns, K. C. Adaptive numerical competency in a food-hoarding

548 songbird. Proc. R. Soc. B Biol. Sci. 275(1649), 2373-2379 (2008).

549 https://doi.org/10.1098/rspb.2008.0702

550 Hussar, C. R. \& Pasternak, T. Flexibility of sensory representations in prefrontal

551 cortex depends on cell type. Neuron 64(5), 730-743 (2009).

552 https://doi.org/10.1016/j.neuron.2009.11.018

553 Izard, V., Sann, C., Spelke, E. S. \& Streri, A. Newborn infants perceive abstract

554 numbers. Proc. Natl. Acad. Sci. USA 106(25), $10382-10385 \quad$ (2009).

555 https://doi.org/10.1073/pnas.0812142106

556 Kim, G., Jang, J., Baek, S., Song, M. \& Paik, S. B. Visual number sense in untrained

557 deep neural networks. Sci. Adv.7(1), eabd6127 (2021). DOI:

$558 \quad 10.1126 /$ sciadv.abd6127

559 Kutter, E. F., Bostroem, J., Elger, C. E., Mormann, F. \& Nieder, A. Single neurons in

560 the human brain encode numbers. Neuron 100(3), $\quad 753-761 \quad$ (2018).

561 https://doi.org/10.1016/j.neuron.2018.08.036

562 Lorenzi, E., Perrino, M. \& Vallortigara, G. Numerosities and Other Magnitudes in the

563 Brains: A Comparative View. Front. Psychol. 12, $1104 \quad$ (2021).

564 https://doi.org/10.3389/fpsyg.2021.641994

565 Lyon, B. E. Egg recognition and counting reduce costs of avian conspecific brood

566 parasitism. Nature 422, 495-499 (2003). https://doi.org/10.1038/nature01505 
567 Messina, A., Potrich, D., Schiona, I., Sovrano, V.A., Fraser, S.E., Brennan, C.H. \&

568 Vallortigara, G. Neurons in the dorso-central division of zebrafish pallium respond to 569 change in visual numerosity. Cereb. Cortex., bhab218 (2021). DOI:

\section{$570 \quad 10.1093 /$ cercor/bhab218}

571 Nasr, K., Viswanathan, P. \& Nieder, A. Number detectors spontaneously emerge in a 572 deep neural network designed for visual object recognition. Sci. Adv. 5(5), eaav7903 573 (2019). DOI: 10.1126/sciadv.aav7903

574 Nieder, A. Supramodal numerosity selectivity of neurons in primate prefrontal and 575 posterior parietal cortices. Proc. Natl. Acad. Sci. USA 109(29), 11860-11865 (2012). 576 https://doi.org/10.1073/pnas.1204580109

577 Nieder, A. The neuronal code for number. Nat. Rev. Neurosci. 17(6), 366-382 578 (2016). https://doi.org/10.1038/nrn.2016.40

579 Nieder, A. A brain for numbers: the biology of the number instinct. (MIT press, 2019).

580 Nieder, A., Freedman, D. J. \& Miller, E. K. Representation of the quantity of visual 581 items in the primate prefrontal cortex. Science 297(5587), 1708-1711 (2002). DOI: $582 \quad 10.1126 /$ science.1072493

583 Nieder, A., Wagener, L. \& Rinnert, P. A neural correlate of sensory consciousness in 584 a corvid bird. Science 369(6511), 1626-1629 (2020). DOI: 10.1126/science.abb1447

585 Olkowicz, S., Kocourek, M., Lučan, R.K., Porteš, M., Fitch, W.T., Herculano-Houzel, 586 S. \& Němec, P. Birds have primate-like numbers of neurons in the forebrain. Proc. 587 Natl. Acad. Sci. USA 113(26), $\quad$ 7255-7260 $\quad$ (2016). 588 https://doi.org/10.1073/pnas. 1517131113 
589 Peirce, J., Gray, J.R., Simpson, S., MacAskill, M., Höchenberger, R., Sogo, H.,

590 Kastman, E. and Lindeløv, J.K. PsychoPy2: Experiments in behavior made 591 easy. Behav. Res. Methods 51(1), 195-203 (2019). https://doi.org/10.3758/s13428592 018-01193-y

593 Potrich, D., Sovrano, V. A., Stancher, G. \& Vallortigara, G. Quantity discrimination by 594 zebrafish (Danio rerio). J. Comp. Psychol. 129(4), $388 \quad$ (2015). 595 https://doi.org/10.1037/com0000012

596 Preuss, S. J., Trivedi, C. A., vom Berg-Maurer, C. M., Ryu, S. \& Bollmann, J. H. 597 Classification of object size in retinotectal microcircuits. Curr. Biol. 24(20), 2376-2385 598 (2014). https://doi.org/10.1016/j.cub.2014.09.012

599 Preuss, T. M. \& Wise, S. P. Evolution of prefrontal cortex. 600 Neuropsychopharmacology 47(1), 3-19 (2022). https://doi.org/10.1038/s41386-021$601 \quad 01076-5$

602 Prum, R.O., Berv, J.S., Dornburg, A., Field, D.J., Townsend, J.P., Lemmon, E.M. \& 603 Lemmon, A.R. A comprehensive phylogeny of birds (Aves) using targeted next604 generation DNA sequencing. Nature 526(7574), $\quad$ 569-573 (2015). 605 https://doi.org/10.1038/nature15697

606 Puelles, L. Comments on the updated tetrapartite pallium model in the mouse and 607 chick, featuring a homologous claustro-insular complex. Brain Behav. Evol. 90(2), 608 171-189 (2017). https://doi.org/10.1159/000479782

609 R Core Team (2020). R: A language and environment for statistical computing. R 610 Foundation for Statistical Computing, Vienna, Austria. URL https://www.R611 project.org/ 
612 Rugani, R., Cavazzana, A., Vallortigara, G. \& Regolin, L. One, two, three, four, or is 613 there something more? Numerical discrimination in day-old domestic chicks. Anim. 614 Cogn. 16(4), 557-564 (2013). DOI 10.1007/s10071-012-0593-8

615 Rugani, R., Fontanari, L., Simoni, E., Regolin, L. \& Vallortigara, G. Arithmetic in 616 newborn chicks. Proc. R. Soc. B Biol. Sci. 276(1666), 2451-2460 (2009). 617 https://doi.org/10.1098/rspb.2009.0044

618 Rugani, R., Regolin, L. \& Vallortigara, G. Discrimination of small numerosities in 619 young chicks. J. Exp. Psychol. Anim. Behav. Process. 34(3), 388 (2008). 620 https://doi.org/10.1037/0097-7403.34.3.388

621 Rugani, R., Vallortigara, G., Priftis, K. \& Regolin, L. Number-space mapping in the 622 newborn chick resembles humans' mental number line. Science 347(6221), 534-536 623 (2015). DOI: 10.1126/science.aaa1379

624 Sawamura, H., Shima, K. \& Tanji, J. Numerical representation for action in the 625 parietal cortex of the monkey. Nature 415(6874), 918-922 (2002). 626 https://doi.org/10.1038/415918a

627 Schmid, K. L. \& Wildsoet, C. F. Assessment of visual acuity and contrast sensitivity 628 in the chick using an optokinetic nystagmus paradigm. Vision Res. 38(17), 26296292634 (1998). https://doi.org/10.1016/S0042-6989(97)00446-X

630 Stacho, M., Herold, C., Rook, N., Wagner, H., Axer, M., Amunts, K. \& Güntürkün, O. 631 A cortex-like canonical circuit in the avian forebrain. Science 369(6511), (2020). DOI: 
633 Stancher, G., Rugani, R., Regolin, L. \& Vallortigara, G. Numerical discrimination by

634 frogs (Bombina orientalis). Anim. Cogn. 18(1), 219-229 (2015). DOI 10.1007/s10071-

$635 \quad 014-0791-7$

636 Stevens, S. S. To honor Fechner and repeal his law. Science 133(3446), 80-86 637 (1961).

638 Striedter, G. F. \& Northcutt, R. G. Brains through time: a natural history of 639 vertebrates. (Oxford University Press, 2019).

640 Templeton, C. N., Greene, E. \& Davis, K. Allometry of alarm calls: black-capped 641 chickadees encode information about predator size. Science 308, 1934-1937 642 (2005). DOI: 10.1126/science.1108841

643 Veit, L. \& Nieder, A. Abstract rule neurons in the endbrain support intelligent 644 behaviour in corvid songbirds. Nat. Commun. 4(1), 1-11 (2013). 645 https://doi.org/10.1038/ncomms3878

646 Veit, L., Pidpruzhnykova, G. \& Nieder, A. Associative learning rapidly establishes 647 neuronal representations of upcoming behavioral choices in crows. Proc. Natl. Acad. 648 Sci. USA 112(49), 15208-15213 (2015). https://doi.org/10.1073/pnas.1509760112

649 Viswanathan, P. \& Nieder, A. Neuronal correlates of a visual "sense of number" in 650 primate parietal and prefrontal cortices. Proc. Natl. Acad. Sci. USA 110(27), 1118765111192 (2013). https://doi.org/10.1073/pnas.1308141110

652 von Eugen, K., Tabrik, S., Güntürkün, O. \& Ströckens, F. A comparative analysis of 653 the dopaminergic innervation of the executive caudal nidopallium in pigeon, chicken, 654 zebra finch, and carrion crow. J. Comp. Neurol.528(17), 2929-2955 (2020). 655 https://doi.org/10.1002/cne.24878 
656 Wagener, L., Loconsole, M., Ditz, H. M. \& Nieder, A. Neurons in the endbrain of

657 numerically naive crows spontaneously encode visual numerosity. Curr. Biol. 28(7),

658 1090-1094 (2018). https://doi.org/10.1016/j.cub.2018.02.023

659 Ward, C. \& Smuts, B. Quantity-based judgments in the domestic dog (Canis lupus

660 familiaris). Anim. Cogn. 10, 71-80 (2007). DOI 10.1007/s10071-006-0042-7

661 Zanon, M., Potrich, D., Bortot, M. \& Vallortigara, G. Towards a standardization of 662 non-symbolic numerical experiments: GeNEsIS, a flexible and user-friendly tool to 663 generate controlled stimuli. Behav. Res. Methods, 1-12 (2021). 664 https://doi.org/10.3758/s13428-021-01580-y

665 Zappia, J. V. \& Rogers, L. J. Sex differences and reversal of brain asymmetry by 666 testosterone in chickens. Behav. Brain Res. 23(3), 261-267 (1987).

667 https://doi.org/10.1016/0166-4328(87)90026-X 


\section{Figures}

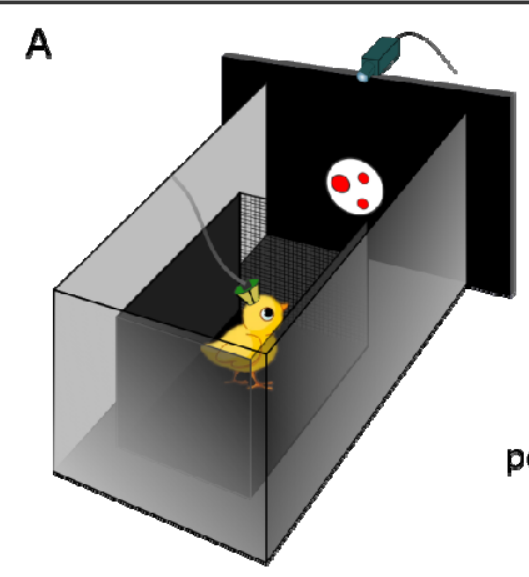

B perimeter

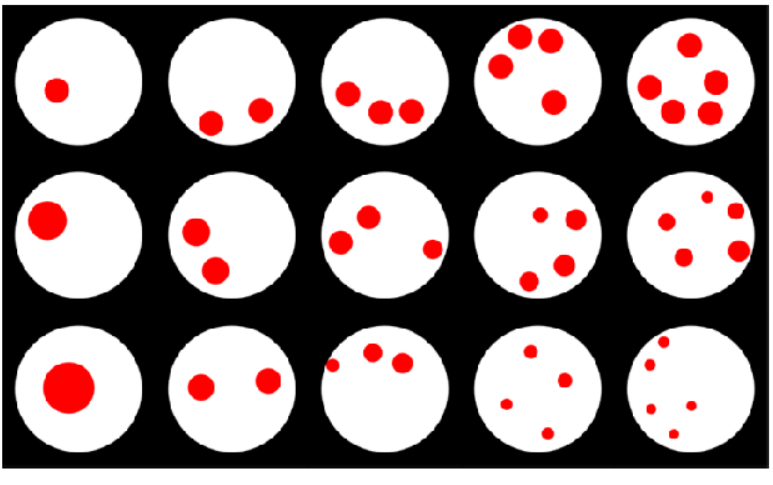

679 Figure 1. Experimental design. (A) Schematic drawing of the experimental setup.

680 Young chicks were placed in a small wooden box in front of the screen, where

681 numerical stimuli appeared. They were trained to pay attention to the stimuli without

682 any further discrimination between different numerosities. (B) Examples of different

683 types of numerosity stimuli that we presented by every neural recording: "radius-

684 fixed", "area-fixed", and "perimeter-fixed". 


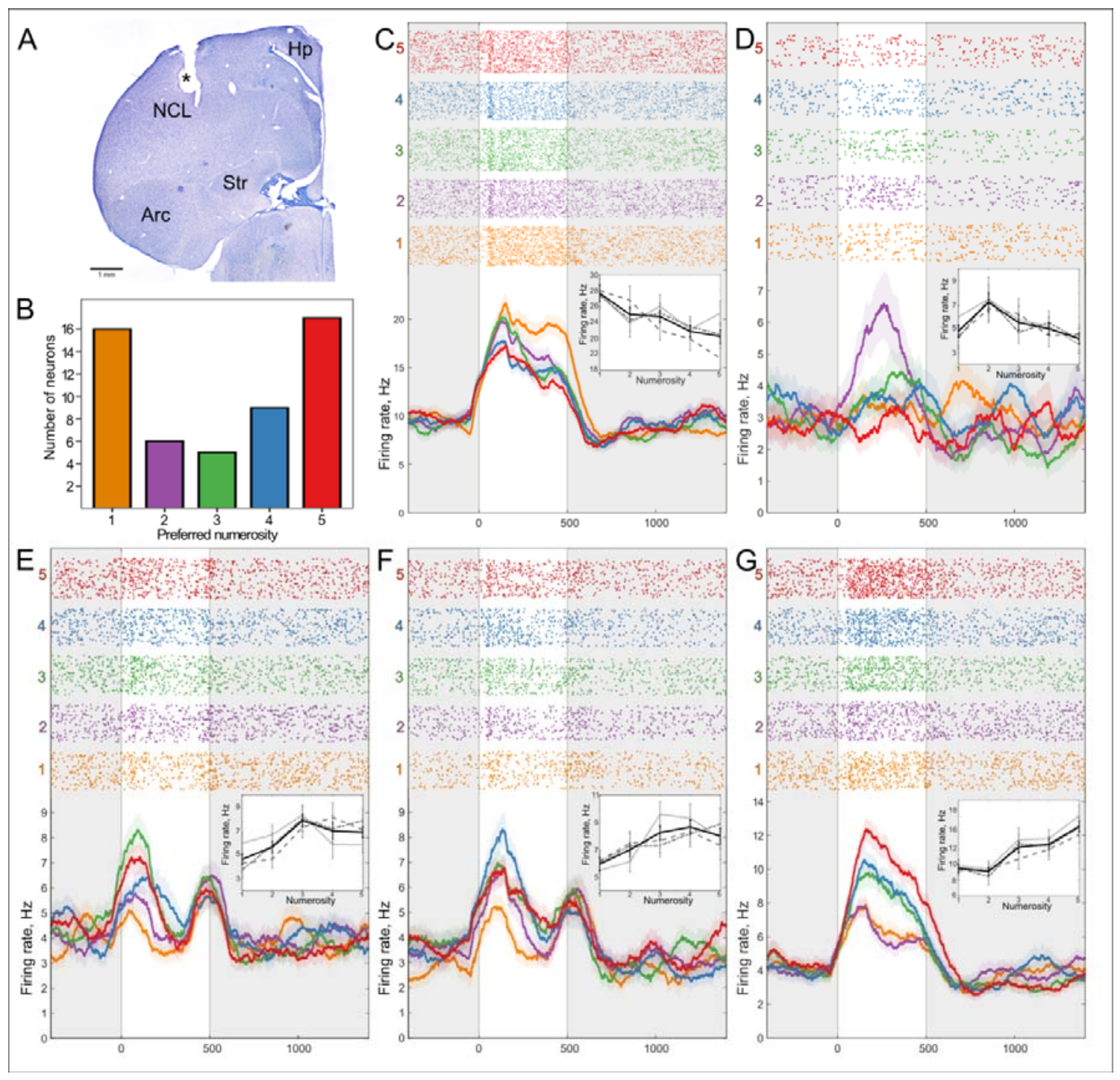

Figure 2. Neurons in the NCL of chicks responding to numerosity. (A) An exemplary coronal section of the chicken forebrain showing the recording site in the NCL (electrolytic lesion is marked by an asterisk). Arc: arcopallium, Hp: hippocampus, NCL: caudolateral nidopallium, Str: striatum. (B) Distribution of neurons that preferred each numerosity stimulus. Examples of neurons that were tuned to 692 numerosity $1(C), 2(D), 3(E), 4(F)$, or $5(G)$. Top: raster plots representing neural activity, where each line corresponds to one trial and each dot corresponds to a spike. Trials are grouped by numerosity. The $500-\mathrm{ms}$ duration of the stimulus is marked by a transparent window. Bottom: averaged spike density functions 
696 (smoothed by a 100 ms Gaussian kernel; SEM is plotted as a shaded area along the

697 lines). Insert: average firing rate in response to numerosities of each stimulus type.

698 Grey dotted line corresponds to "radius-fixed", dashed line - to "perimeter-fixed", dot-

699 dashed line - to "area-fixed" stimuli, and black solid line - to an average. Error bars

700 correspond to SEM.

701

702

703

704

705

706

707

708

709

710

711

712 

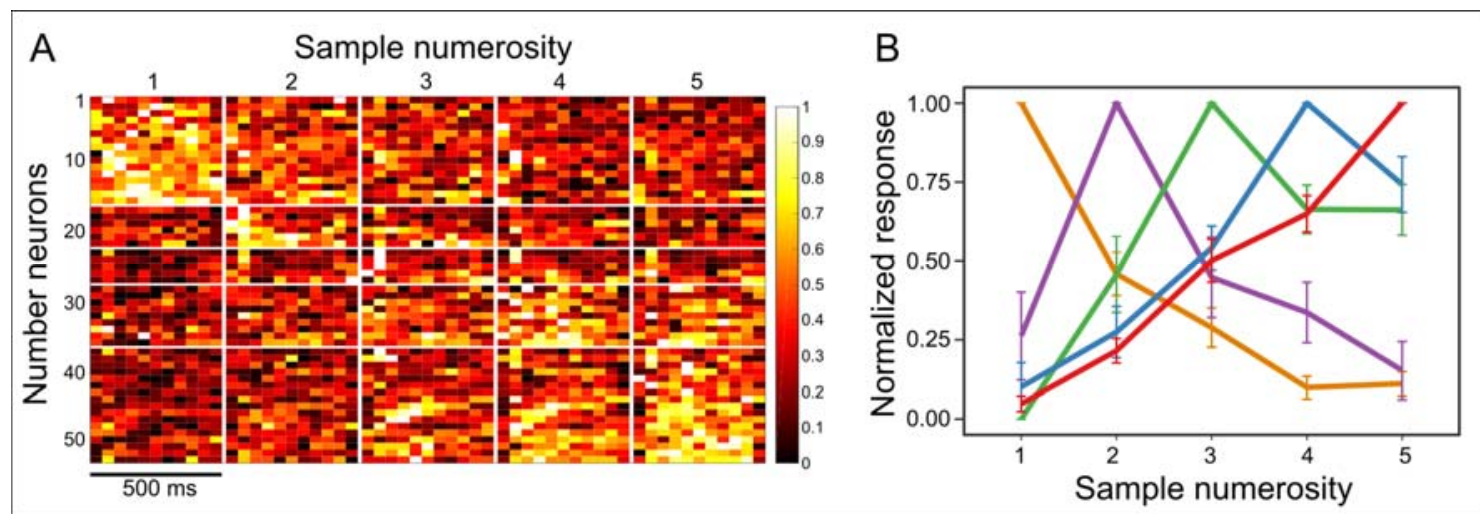

714 Figure 3. (A) Neural response of all recorded number neurons to numerosity stimuli.

715 Heatmap values represent the mean firing rate during the stimulus presentation

716 (binned by $50-\mathrm{ms}$ ), normalised $[0,1]$ for the corresponding neuron in each row.

717 Values are further grouped by the numerosity stimuli from 1 to 5 (vertical white lines),

718 and by the numerical preference of recorded neurons (horizontal white lines) from

719 neurons that preferred numerosity 1 (top) to neurons that preferred numerosity 5

720 (bottom). (B) Average tuning curves of numerosity selective neurons. The neural

721 activity of neurons is first normalised [0 = response to the least-preferred numerosity,

7221 = response to the most-preferred numerosity] and then grouped by their most

723 preferred numerosity. Error bars correspond to SEM. 
bioRxiv preprint doi: https://doi.org/10.1101/2022.01.21.475044; this version posted January 21, 2022. The copyright holder for this preprint (which was not certified by peer review) is the author/funder, who has granted bioRxiv a license to display the preprint in perpetuity. It is made available under aCC-BY-NC-ND 4.0 International license.
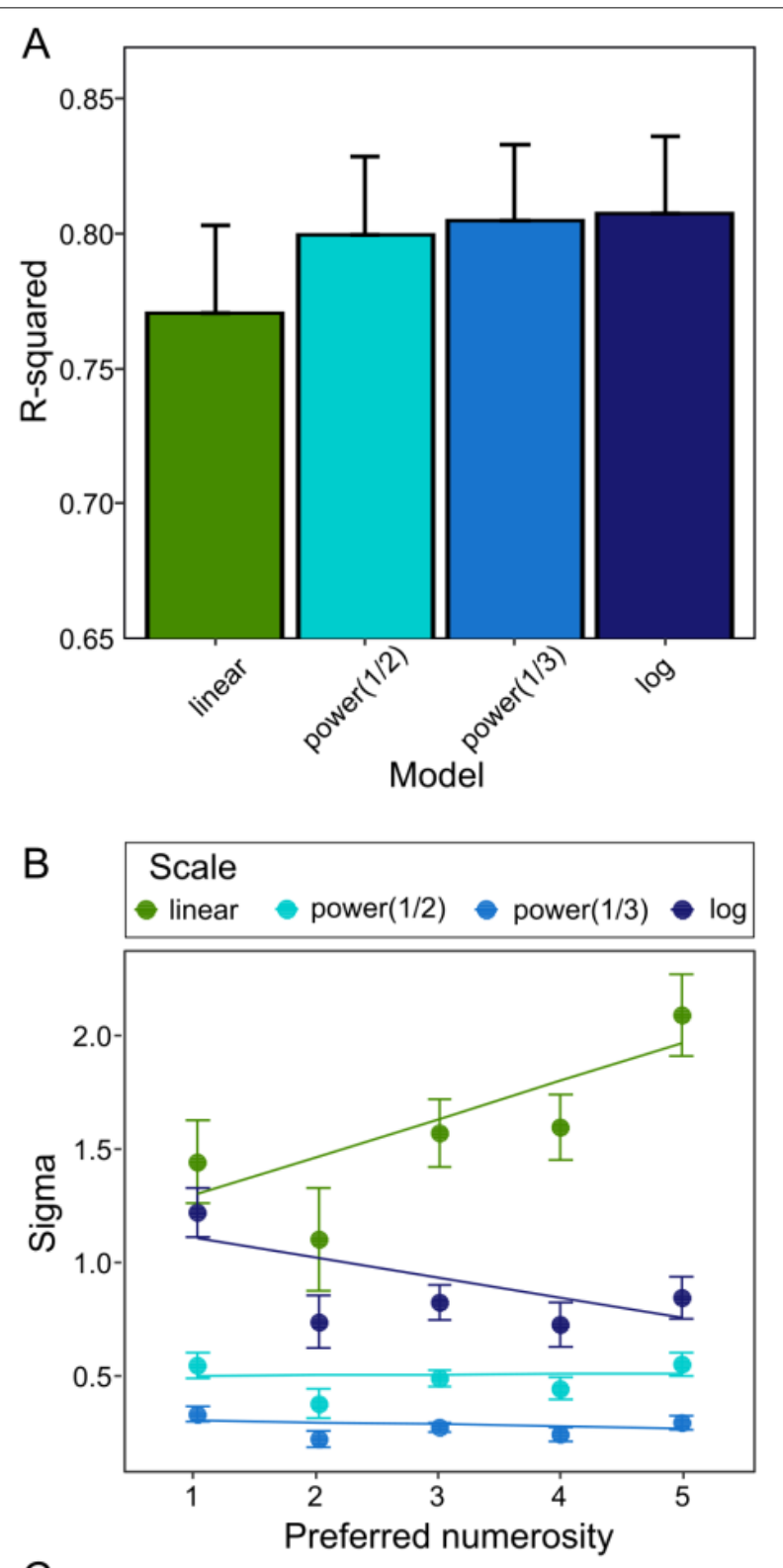

C

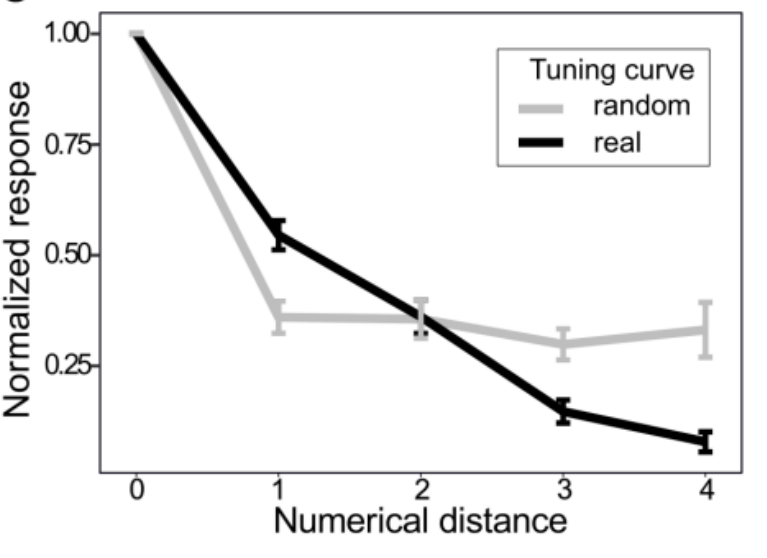


725 Figure 4. (A-B) Comparison of different scaling schemes for the tuning curves. (A) R-

726 squared, a measure of goodness-of-fit reflects symmetry of the tuning curves plotted

727 on the four different scales. The tuning curves of number neurons become more

728 symmetric when plotted on the non-linear scale. (B) Sigma of the Gaussian fit for

729 neurons preferring different numerosities. When plotted on the linear scale, the

730 tuning curves become wider with increased numerosity. Error bars correspond to

731 SEM. (C) Averaged normalised activity of all numerosity selective neurons compared

732 to the random tuning curve (see Materials and methods section for details). The

733 neural activity was normalised [0 = response to the least-preferred numerosity, $1=$

734 response to the most-preferred numerosity] and then plotted as a function of

735 absolute numerical distance from the most preferred numerosity. Neural response of

736 numerosity selective neurons (black line) gradually decreased with the numerical

737 distance. The slope of this tuning curve is notably different from the random tuning

738 curve (grey line) of false-positive neurons obtained by random shuffling of trials.

739 Error bars correspond to SEM. 


\begin{tabular}{cccc}
\hline Preferred & \multicolumn{2}{c}{ ANOVA (Firing rate $\sim$ Stimulus type * Numerosity) } \\
\cline { 2 - 4 } numerosity & Numerosity & Stimulus type & Interaction \\
\cline { 2 - 4 } & $F(4,251)=5.7273, p<0.001$ & $F(2,251)=0.9275, p=0.396$ & $F(8,251)=1.2411, p=0.273$ \\
num1 & $F(4,253)=3.5881, p=0.007$ & $F(2,253)=0.2355, p=0.79$ & $F(8,253)=0.454, p=0.887$ \\
num3 & $F(4,496)=5.8789, p<0.001$ & $F(2,496)=0.1179, p=0.889$ & $F(8,496)=1.4538, p=0.173$ \\
num4 & $F(4,308)=4.2385, p=0.002$ & $F(2,308)=0.1141, p=0.892$ & $F(8,308)=0.9124, p=0.506$ \\
num5 & $F(4,355)=13.4938, p<0.001$ & $F(2,355)=1.832, p=0.162$ & $F(8,355)=0.3219, p=0.958$
\end{tabular}

750 Table 1. Results of the two-way ANOVA for five example number neurons shown in

751 the Figure $2(\mathrm{C}-\mathrm{G})$. Preferred numerosity: numerosity eliciting the strongest

752 response. ANOVA results (F-statistics and p-value) for the factor "stimulus type"

753 ("radius-fixed", "area-fixed", "perimeter-fixed"), "numerosity" (numerosity 1 to 5), or

754 interaction between them.

755

\begin{tabular}{ccccccc}
\hline \multicolumn{2}{c}{ Summary } & \multicolumn{5}{c}{ Number of neurons } \\
\hline Sum & Group & num1 & num2 & num3 & num4 & num5 \\
16 & num1 & & 6 & 9 & 12 & 14 \\
6 & num2 & 4 & & 2 & 1 & 3 \\
5 & num3 & 5 & 1 & & 0 & 0 \\
9 & num4 & 6 & 2 & 1 & & 1 \\
17 & num5 & 15 & 12 & 6 & 1 & \\
\hline
\end{tabular}

757 Table 2. The summary of the post hoc analysis. For each group based on their

758 preferred numerosity we calculated the number of neurons that showed significant

759 difference between the most-preferred and the given numerosity. 


\section{Supplementary information}

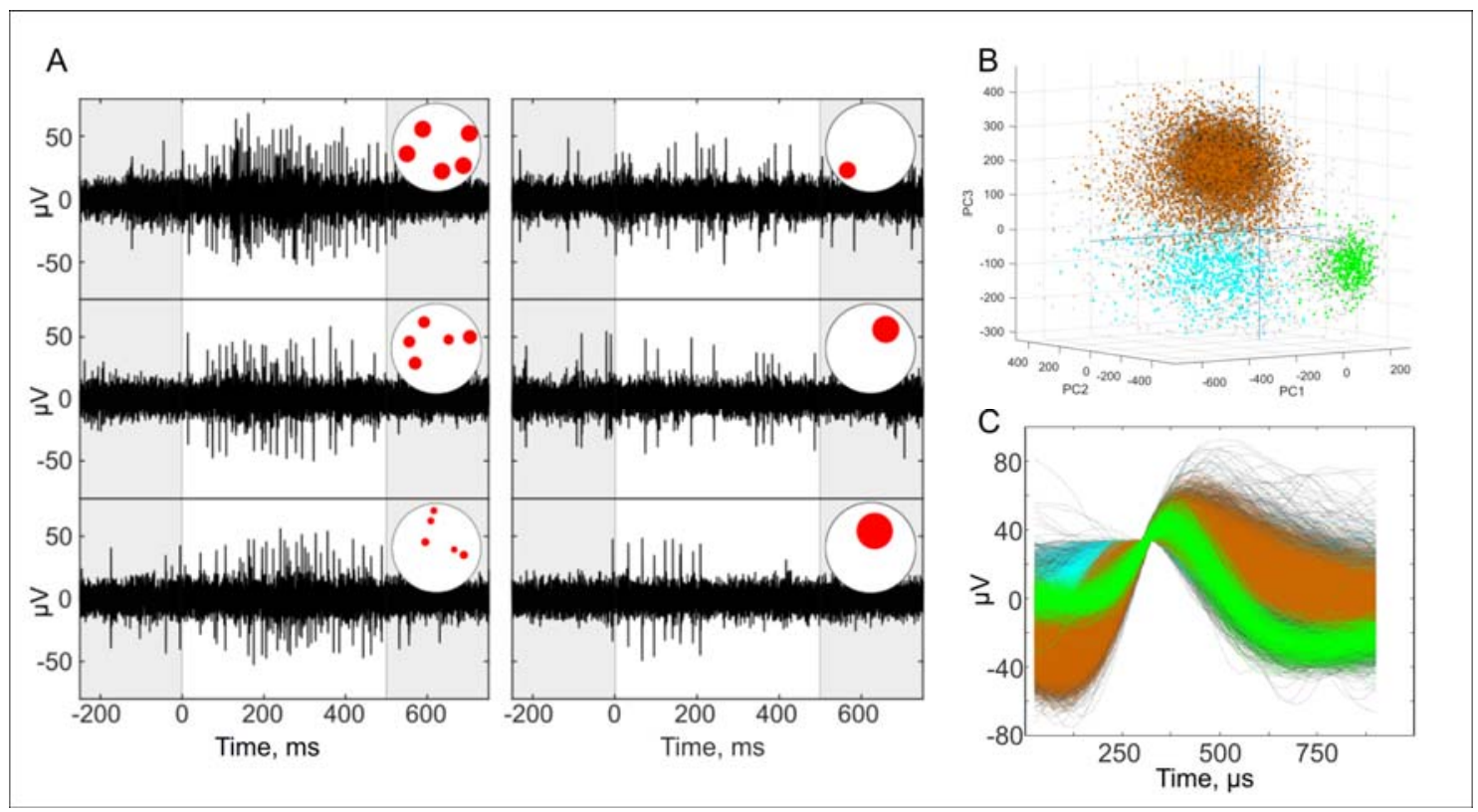

763 Figure S1. (A) Example of raw spike trains: electrical signal is shown after the highpass filter was applied. Examples of single trials, representing neural response of the

unit to numerosity 5 (left column), irrespective of the stimulus appearance (top: radius-fixed, middle: area-fixed, bottom: perimeter-fixed). Note the decreasing neural response to the numerosity 1 (right column).

(B) The PCA clustering of the corresponding recording with waveforms of different units shown by different colours.

769 The waveforms of the number-responsive unit are shown in orange, unsorted waveforms are shown in grey. (C) Spike waveforms of corresponding units isolated from the recording of one electrode. 
bioRxiv preprint doi: https://doi.orq/10.1101/2022.01.21.475044: this version posted Januarv 21. 2022. The copvright holder for this preprint (which was not certified by peer review) is the author/funder, who has granted bioRxiv a license to display the preprint in perpetuity. It is made available under aCC-BY-NC-ND 4.0 International license.

\begin{tabular}{|c|c|c|c|c|}
\hline \multirow{2}{*}{ Unit } & \multirow{2}{*}{$\begin{array}{c}\text { Preferred } \\
\text { numerosity }\end{array}$} & \multicolumn{3}{|c|}{ ANOVA (Firing rate $\sim$ Stimulus type * Numerosity) } \\
\hline & & Numerosity & Stimulus type & Interaction \\
\hline 1 & num1 & $F(4,546)=4.0327, p=0.003$ & $F(2,546)=0.5228, p=0.593$ & $F(8,546)=1.4242, p=0.183$ \\
\hline 2 & num1 & $F(4,197)=3.5672, p=0.008$ & $F(2,197)=0.4656, p=0.628$ & $F(8,197)=0.8763, p=0.537$ \\
\hline 3 & num1 & $F(4,202)=4.0268, p=0.004$ & $F(2,202)=1.7732, p=0.172$ & $F(8,202)=1.1967, p=0.303$ \\
\hline 4 & num1 & $F(4,565)=4.2588, p=0.002$ & $F(2,565)=0.0012, p=0.999$ & $F(8,565)=0.7852, p=0.616$ \\
\hline 5 & num1 & $F(4,270)=5.3565, p=0$ & $F(2,270)=1.2038, p=0.302$ & $F(8,270)=0.9519, p=0.474$ \\
\hline 6 & num1 & $F(4,270)=5.4507, p=0$ & $F(2,270)=0.0475, p=0.954$ & $F(8,270)=0.7056, p=0.687$ \\
\hline 7 & num1 & $F(4,239)=3.7861, p=0.005$ & $F(2,239)=0.4114, p=0.663$ & $F(8,239)=1.6597, p=0.109$ \\
\hline 8 & num1 & $F(4,419)=4.9175, p=0.001$ & $F(2,419)=1.0111, p=0.365$ & $F(8,419)=1.0988, p=0.363$ \\
\hline 9 & num1 & $F(4,399)=7.4226, p=0$ & $F(2,399)=2.8675, p=0.058$ & $F(8,399)=2.1769, p=0.028$ \\
\hline 10 & num1 & $F(4,366)=4.2597, p=0.002$ & $F(2,366)=0.9588, p=0.384$ & $F(8,366)=1.4233, p=0.185$ \\
\hline 11 & num1 & $F(4,364)=6.4075, p=0$ & $F(2,364)=0.2249, p=0.799$ & $F(8,364)=1.2606, p=0.263$ \\
\hline 12 & num1 & $F(4,490)=3.9053, p=0.004$ & $F(2,490)=1.1902, p=0.305$ & $F(8,490)=0.6286, p=0.754$ \\
\hline 13 & num1 & $F(4,251)=5.7273, p=0$ & $F(2,251)=0.9275, p=0.396$ & $F(8,251)=1.2411, p=0.273$ \\
\hline 14 & num1 & $F(4,186)=3.5545, p=0.008$ & $F(2,186)=1.1998, p=0.304$ & $F(8,186)=0.6334, p=0.749$ \\
\hline 15 & num1 & $F(4,233)=4.4063, p=0.002$ & $F(2,233)=1.138, p=0.322$ & $F(8,233)=0.3761, p=0.933$ \\
\hline 16 & num1 & $F(4,316)=3.6533, p=0.006$ & $F(2,316)=1.4135, p=0.245$ & $F(8,316)=0.8235, p=0.582$ \\
\hline 17 & num2 & $F(4,177)=4.5348, p=0.002$ & $F(2,177)=0.2742, p=0.761$ & $F(8,177)=0.6772, p=0.711$ \\
\hline 18 & num2 & $F(4,176)=4.1623, p=0.003$ & $F(2,176)=0.8853, p=0.414$ & $F(8,176)=0.8814, p=0.533$ \\
\hline 19 & num2 & $F(4,147)=3.6633, p=0.007$ & $F(2,147)=0.929, p=0.397$ & $F(8,147)=0.5183, p=0.841$ \\
\hline 20 & num2 & $F(4,145)=3.7461, p=0.006$ & $F(2,145)=0.514, p=0.599$ & $F(8,145)=0.5375, p=0.827$ \\
\hline 21 & num2 & $F(4,298)=3.4934, p=0.008$ & $F(2,298)=0.7184, p=0.488$ & $F(8,298)=1.0979, p=0.364$ \\
\hline 22 & num2 & $F(4,253)=3.5881, p=0.007$ & $F(2,253)=0.2355, p=0.79$ & $F(8,253)=0.454, p=0.887$ \\
\hline 23 & num3 & $F(4,213)=3.4856, p=0.009$ & $F(2,213)=0.6473, p=0.524$ & $F(8,213)=0.5014, p=0.854$ \\
\hline 24 & num3 & $F(4,184)=3.6183, p=0.007$ & $F(2,184)=0.5629, p=0.571$ & $F(8,184)=0.6608, p=0.725$ \\
\hline 25 & num3 & $F(4,472)=5.1242, p=0$ & $F(2,472)=2.8061, p=0.062$ & $F(8,472)=0.809, p=0.595$ \\
\hline 26 & num3 & $F(4,410)=3.7563, p=0.005$ & $F(2,410)=4.5228, p=0.011$ & $F(8,410)=1.3146, p=0.233$ \\
\hline 27 & num3 & $F(4,496)=5.8789, p=0$ & $F(2,496)=0.1179, p=0.889$ & $F(8,496)=1.4538, p=0.173$ \\
\hline 28 & num4 & $F(4,176)=3.926, p=0.004$ & $F(2,176)=1.1196, p=0.329$ & $F(8,176)=0.6298, p=0.752$ \\
\hline 29 & num4 & $F(4,178)=3.9175, p=0.004$ & $F(2,178)=0.4244, p=0.655$ & $F(8,178)=0.6897, p=0.7$ \\
\hline 30 & num4 & $F(4,267)=4.5852, p=0.001$ & $F(2,267)=0.6043, p=0.547$ & $F(8,267)=0.8609, p=0.55$ \\
\hline 31 & num4 & $F(4,371)=3.6503, p=0.006$ & $F(2,371)=1.9928, p=0.138$ & $F(8,371)=0.5894, p=0.787$ \\
\hline 32 & num4 & $F(4,430)=3.4615, p=0.008$ & $F(2,430)=0.3739, p=0.688$ & $F(8,430)=0.513, p=0.847$ \\
\hline
\end{tabular}




\begin{tabular}{|c|c|c|c|c|}
\hline 33 & num4 & $F(4,420)=4.4972, p=0.001$ & $F(2,420)=1.1432, p=0.32$ & $F(8,420)=1.1135, p=0.353$ \\
\hline 34 & num4 & $F(4,459)=6.5488, p=0$ & $F(2,459)=2.8234, p=0.06$ & $F(8,459)=1.3186, p=0.232$ \\
\hline 35 & num4 & $F(4,333)=3.4399, p=0.009$ & $F(2,333)=3.3226, p=0.037$ & $F(8,333)=1.5331, p=0.145$ \\
\hline 36 & num4 & $F(4,308)=4.2385, p=0.002$ & $F(2,308)=0.1141, p=0.892$ & $F(8,308)=0.9124, p=0.506$ \\
\hline 37 & num5 & $F(4,323)=5.0184, p=0.001$ & $F(2,323)=1.9782, p=0.14$ & $F(8,323)=0.3722, p=0.935$ \\
\hline 38 & num5 & $F(4,188)=3.5707, p=0.008$ & $F(2,188)=1.1655, p=0.314$ & $F(8,188)=0.5456, p=0.821$ \\
\hline 39 & num5 & $F(4,307)=5.1489, p=0$ & $F(2,307)=0.0322, p=0.968$ & $F(8,307)=0.4935, p=0.861$ \\
\hline 40 & num5 & $F(4,260)=5.1953, p=0$ & $F(2,260)=0.945, p=0.39$ & $F(8,260)=0.682, p=0.707$ \\
\hline 41 & num5 & $F(4,234)=4.65, p=0.001$ & $F(2,234)=0.7971, p=0.452$ & $F(8,234)=0.8709, p=0.542$ \\
\hline 42 & num5 & $F(4,232)=3.7216, p=0.006$ & $F(2,232)=0.0802, p=0.923$ & $F(8,232)=1.3969, p=0.199$ \\
\hline 43 & num5 & $F(4,484)=3.6509, p=0.006$ & $F(2,484)=0.1205, p=0.887$ & $F(8,484)=0.7686, p=0.631$ \\
\hline 44 & num5 & $F(4,458)=12.8202, p=0$ & $F(2,458)=0.7574, p=0.469$ & $F(8,458)=0.4219, p=0.908$ \\
\hline 45 & num5 & $F(4,192)=4.4432, p=0.002$ & $F(2,192)=2.0198, p=0.135$ & $F(8,192)=1.8229, p=0.075$ \\
\hline 46 & num5 & $F(4,415)=3.8044, p=0.005$ & $F(2,415)=1.618, p=0.2$ & $F(8,415)=0.5316, p=0.832$ \\
\hline 47 & num5 & $F(4,685)=14.1207, p=0$ & $F(2,685)=1.9687, p=0.141$ & $F(8,685)=1.4958, p=0.156$ \\
\hline 48 & num5 & $F(4,371)=6.7422, p=0$ & $F(2,371)=0.6683, p=0.513$ & $F(8,371)=1.6257, p=0.115$ \\
\hline 49 & num5 & $F(4,359)=4.1619, p=0.003$ & $F(2,359)=0.1355, p=0.873$ & $F(8,359)=0.4252, p=0.906$ \\
\hline 50 & num5 & $F(4,355)=13.4938, p=0$ & $F(2,355)=1.832, p=0.162$ & $F(8,355)=0.3219, p=0.958$ \\
\hline 51 & num5 & $F(4,354)=6.4972, p=0$ & $F(2,354)=0.6433, p=0.526$ & $F(8,354)=0.9821, p=0.45$ \\
\hline 52 & num5 & $F(4,288)=3.3897, p=0.01$ & $F(2,288)=2.1069, p=0.123$ & $F(8,288)=1.2445, p=0.273$ \\
\hline 53 & num5 & $F(4,460)=5.4865, p=0$ & $F(2,460)=1.4027, p=0.247$ & $F(8,460)=0.7622, p=0.636$ \\
\hline
\end{tabular}

776 Table S1. Summary of the two-way ANOVA for every recorded unit. Unit: id of the recorded unit. Preferred numerosity: numerosity stimulus that elicited the strongest response in the corresponding unit. ANOVA results (F-statistics and p-value) are 779 summarized for the factor "Stimulus type" (radius-fixed, area-fixed, perimeter-fixed), 780 "Numerosity" (numerosity "one" to "five"), or interaction between them. 


\begin{tabular}{|c|c|c|c|c|c|c|}
\hline \multirow{2}{*}{ Unit } & \multirow{2}{*}{$\begin{array}{c}\text { Preferred } \\
\text { numerosity }\end{array}$} & \multicolumn{5}{|c|}{ Post hoc analysis, p-value } \\
\hline & & num1 & num2 & num3 & num4 & num5 \\
\hline 1 & num1 & & 0.016 & 0.022 & 0.019 & 0.007 \\
\hline 2 & num1 & & 0.081 & 0.019 & 0.011 & 0.021 \\
\hline 3 & num1 & & 0.988 & 0.903 & 0.303 & 0.003 \\
\hline 4 & num1 & & 0.974 & 0.315 & 0.002 & 0.209 \\
\hline 5 & num1 & & 0.890 & 0.650 & 0.000 & 0.027 \\
\hline 6 & num1 & & 0.176 & 0.063 & 0.001 & 0.001 \\
\hline 7 & num1 & & 0.035 & 0.048 & 0.006 & 0.020 \\
\hline 8 & num1 & & 0.031 & 0.001 & 0.001 & 0.023 \\
\hline 9 & num1 & & 0.788 & 0.174 & 0.000 & 0.000 \\
\hline 10 & num1 & & 0.465 & 0.002 & 0.009 & 0.169 \\
\hline 11 & num1 & & 0.033 & 0.000 & 0.000 & 0.001 \\
\hline 12 & num1 & & 0.036 & 0.013 & 0.090 & 0.004 \\
\hline 13 & num1 & & 0.221 & 0.120 & 0.001 & 0.000 \\
\hline 14 & num1 & & 0.147 & 0.142 & 0.061 & 0.004 \\
\hline 15 & num1 & & 0.031 & 0.002 & 0.084 & 0.004 \\
\hline 16 & num1 & & 0.304 & 0.048 & 0.008 & 0.014 \\
\hline 17 & num2 & 0.447 & & 0.986 & 0.362 & 0.001 \\
\hline 18 & num2 & 0.001 & & 0.027 & 0.025 & 0.206 \\
\hline 19 & num2 & 0.023 & & 0.945 & 0.065 & 0.063 \\
\hline 20 & num2 & 0.013 & & 0.017 & 0.826 & 0.608 \\
\hline 21 & num2 & 0.973 & & 0.181 & 0.125 & 0.011 \\
\hline 22 & num2 & 0.040 & & 0.312 & 0.075 & 0.004 \\
\hline 23 & num3 & 0.005 & 0.807 & & 0.760 & 0.123 \\
\hline 24 & num3 & 0.006 & 0.094 & & 0.504 & 0.899 \\
\hline 25 & num3 & 0.001 & 0.913 & & 0.998 & 0.796 \\
\hline 26 & num3 & 0.005 & 0.059 & & 0.370 & 0.857 \\
\hline 27 & num3 & 0.000 & 0.024 & & 0.798 & 0.683 \\
\hline 28 & num4 & 0.003 & 0.282 & 0.120 & & 0.016 \\
\hline 29 & num4 & 0.020 & 0.051 & 0.213 & & 0.999 \\
\hline 30 & num4 & 0.710 & 0.001 & 0.028 & & 0.103 \\
\hline 31 & num4 & 0.007 & 0.369 & 0.813 & & 0.998 \\
\hline 32 & num4 & 0.059 & 0.059 & 0.936 & & 0.999 \\
\hline 33 & num4 & 0.065 & 0.007 & 0.864 & & 1.000 \\
\hline 34 & num4 & 0.000 & 0.092 & 0.419 & & 0.968 \\
\hline 35 & num4 & 0.004 & 0.628 & 0.616 & & 0.850 \\
\hline 36 & num4 & 0.003 & 0.194 & 0.981 & & 0.928 \\
\hline 37 & num5 & 0.002 & 0.002 & 0.007 & 0.054 & \\
\hline 38 & num5 & 0.022 & 0.287 & 0.995 & 0.999 & \\
\hline 39 & num5 & 0.000 & 0.148 & 0.653 & 0.803 & \\
\hline 40 & num5 & 0.000 & 0.026 & 0.525 & 0.214 & \\
\hline 41 & num5 & 0.001 & 0.021 & 0.226 & 0.012 & \\
\hline 42 & num5 & 0.008 & 0.150 & 0.010 & 0.431 & \\
\hline 43 & num5 & 0.004 & 0.097 & 0.111 & 0.725 & \\
\hline 44 & num5 & 0.000 & 0.000 & 0.750 & 0.851 & \\
\hline 45 & num5 & 0.015 & 0.032 & 0.001 & 0.080 & \\
\hline 46 & num5 & 0.070 & 0.002 & 0.428 & 0.069 & \\
\hline
\end{tabular}




\begin{tabular}{llllll}
\hline 47 & num5 & $\mathbf{0 . 0 0 0}$ & $\mathbf{0 . 0 0 0}$ & $\mathbf{0 . 0 1 6}$ & 0.868 \\
48 & num5 & $\mathbf{0 . 0 0 0}$ & $\mathbf{0 . 0 0 3}$ & 0.251 & 0.901 \\
49 & num5 & $\mathbf{0 . 0 0 3}$ & 0.394 & 0.940 & 0.997 \\
50 & num5 & $\mathbf{0 . 0 0 0}$ & $\mathbf{0 . 0 0 0}$ & $\mathbf{0 . 0 3 4}$ & 0.078 \\
51 & num5 & $\mathbf{0 . 0 0 0}$ & $\mathbf{0 . 0 0 9}$ & $\mathbf{0 . 0 1 4}$ & 0.801 \\
52 & num5 & 0.074 & $\mathbf{0 . 0 2 3}$ & 0.988 & 0.798 \\
53 & num5 & $\mathbf{0 . 0 0 6}$ & $\mathbf{0 . 0 0 4}$ & 0.276 & 0.998 \\
\hline
\end{tabular}

783

784 Table S2. The post hoc analysis of the two-way ANOVA based on the Tukey-Kramer

785 method summarising p-values for every pairwise comparison between the most-

786 preferred and other numerosities. Significant $p$-values $<0.05$ are highlighted in bold. 